\title{
COMPLETE INTEGRAL CLOSURE AND STRONGLY DIVISORIAL PRIME IDEALS
}

\author{
VALENTINA BARUCCI, STEFANIA GABELLI, AND MOSHE ROITMAN
}

\begin{abstract}
It is well known that a domain without proper strongly divisorial ideals is completely integrally closed. In this paper we show that a domain without prime strongly divisorial ideals is not necessarily completely integrally closed, although this property holds under some additional assumptions.
\end{abstract}

\section{INTRODUCTION}

The strongly divisorial ideals of a domain $R$ are the nonzero conductors $(R: T)$ for some overring $T$ of $R$. They can be used to describe the complete integral closure $R^{*}$ of $R$; in fact $R^{*}=\bigcup(R: I)$, where $I$ runs throughout the set of the strongly divisorial ideals of $R$. In particular, $R$ is completely integrally closed, (that is $R=R^{*}$ ), if and only if $R$ has no proper strongly divisorial ideals [3. This paper deals with the following question [5, Problem 11, page 461]:

Question. Is a domain $R$ completely integrally closed if $R$ does not have strongly divisorial prime ideals?

We show that in general the answer is negative, and give conditions on $R$ for a positive answer.

The positive results are collected in Section 1. We first prove some criteria useful to establish when a domain having a proper strongly divisorial ideal is forced to have a strongly divisorial prime ideal. We prove that an ideal which is maximal in the set of proper strongly divisorial ideals of a domain is prime (Proposition 1.3) and we give conditions sufficient for a strongly divisorial ideal to have a strongly divisorial minimal prime (Lemmas 1.6 and 1.7).

Applying these results, we show that the answer to the question above is positive for some classes of domains. We prove in Proposition 1.8 that if a domain has a proper strongly divisorial ideal containing a power of its radical and if this radical is an irredundant intersection

1991 Mathematics Subject Classification. Primary: 13A15 ; Secondary: 13G05, $13 \mathrm{~B} 22$.

Key words and phrases. complete integral closure, divisorial ideal. 
of primes, then the domain contains a strongly divisorial prime ideal. We note in Lemma 1.9 that in a seminormal domain each strongly divisorial ideal is radical, and so the first assumption of Proposition 1.8 is always satisfied in the seminormal case. As another consequence of Proposition 1.8, we give a positive answer for any pullback of a Noetherian domain (Theorem 1.11).

We also answer the above question affirmatively for the class of domains with the property that each maximal $t$-ideal is divisorial (Proposition 1.12). This class includes Noetherian, Mori, TV- and pseudovaluation domains. Indeed a domain of this kind without strongly divisorial prime ideals is a Krull domain, as it is pointed out in Theorem 1.13. where there are given several other equivalent conditions in terms of ideals. A domain without strongly divisorial primes satisfying the stronger property that each maximal ideal is invertible is a Dedekind domain (Corollary 1.15).

There are also other important classes of domains for which the question has a positive answer; for example valuation domains (more generally, TP-domains or Prüfer RTP-domains) and one-dimensional seminormal domains with finitely many maximal ideals (Proposition 1.17).

On the other hand we do not know the answer in case of a onedimensional seminormal domain.

In Section 2 we give examples of domains that are not completely integrally closed but do not have strongly divisorial prime ideals. The first two examples are quasilocal and one-dimensional domains whose maximal ideal is not divisorial. The conductor of the complete integral closure is zero in the first example and is nonzero in the second one. These two domains are Archimedean and not seminormal by Proposition [1.17. The third example is an infinite dimensional domain with nonzero conductor in its complete integral closure, and can be chosen to satisfy or not either one of the conditions of being Archimedean or seminormal. In the seminormal case, the conductor is a strongly divisorial radical ideal (Lemma 1.9) such that all its minimal primes are strong but not divisorial.

Throughout this paper, $R$ will denote an integral domain with quotient field $\mathrm{Qf}(R)=K$. For a pair of fractional ideals $I$ and $J$ of a domain $R$ we let $(J: I)$ denote the set $\{x \in K \mid x I \subseteq J\}$ and $\left(J:_{R} I\right)$ denote the set $\{x \in R \mid x I \subseteq J\}$. As usual, we set $I_{v}=(R:(R: I))$ and $I_{t}=\bigcup J_{v}$ with the union taken over all finitely generated fractional ideals $J$ contained in $I$. An ideal $I$ is a $v$-ideal, or divisorial, if $I=I_{v}$, and it is a $t$-ideal if $I=I_{t}$. The $v$ - and the $t$-operations are particular 
*-operations. If $(R: I)=(I: I)$, that is, $I=I(R: I)$, we say that $I$ is a strong ideal or a trace ideal. A strongly divisorial ideal is an ideal that is strong and divisorial and a maximal strongly divisorial ideal is an ideal that is maximal in the set of the proper strongly divisorial ideals. A maximal t-ideal is an ideal that is maximal in the set of the proper integral $t$-ideals. A general reference for systems of ideals and *-operations is [10].

A nonzero element $x \in K$ is said to be almost integral over $R$ if there is a nonzero element $d \in R$ such that $d x^{i} \in R$, for $i \geq 0$. This is equivalent to saying that all the powers of $x$ belong to a finite $R$ module or that they generate a fractional ideal of $R$. The complete integral closure of $R$ in $K$, which we denote by $R^{*}$, is the set of the elements of $K$ that are almost integral over $R$. One says that $R$ is completely integrally closed if $R=R^{*}$.

If an element is integral over $R$, then it is almost integral, and if $R$ is Noetherian the converse holds. Thus, if $R^{\prime}$ denotes the integral closure of $R$ in $K$, one has $R \subseteq R^{\prime} \subseteq R^{*}$, and if $R$ is Noetherian then $R^{\prime}=R^{*}$. It is well known that $R^{*}$ is always integrally closed, but many examples have been given to show that it might not be completely integrally closed. However, $R^{*}$ is completely integrally closed if the conductor $\left(R: R^{*}\right)$ is a nonzero ideal, that is, if $R^{*}$ is contained in a finite $R$-submodule of $K$ (see for example [3] ).

\section{Positive Results}

In this Section we give some criteria which are useful for establishing when a domain having a proper strongly divisorial ideal has a strongly divisorial prime ideal and we apply our criteria to give a positive answer to the Question in the Introduction for some classes of domains.

We start by proving that a maximal strongly divisorial ideal is prime.

Lemma 1.1. Let $R$ be any ring and let $\mathbf{S}$ be a set of proper ideals of $R$. Assume that $\left(I:_{R} x\right) \in \mathbf{S}$ for any $I \in \mathbf{S}$ and nonzero $x \in R \backslash I$. Then an ideal of $R$ that is maximal in $\mathbf{S}$ is prime.

Proof. Let $I$ be an ideal that is maximal in $\mathbf{S}$ and let $x, y \in R$ such that $x y \in I$ and $x \notin I$. We have $I \subseteq\left(I:_{R} x\right) \in \mathbf{S}$. Hence $\left(I:_{R} x\right)=I$ and $y \in\left(I:_{R} x\right)=I$. It follows that $I$ is a prime ideal.

Lemma 1.2. Let $R$ be an integral domain, $I$ a proper strong ideal of $R$ and $x \in(R: I) \backslash I$. Then $\left(I:_{R} x\right)$ is also a proper strong ideal of $R$.

Proof. Clearly $J=\left(I:_{R} x\right)$ is a proper ideal of $R$ containing $I$. Hence $(R: J) \subseteq(R: I)=(I: I)$. Moreover, $(R: J) J x=(I: I)\left(I:_{R} x\right) x \subseteq$ 
I. Thus $(R: J) J \subseteq(I: R x)=J$, which implies that $(R: J)=(J$ : $J)$.

Proposition 1.3. If $I$ is a maximal strongly divisorial ideal of an integral domain $R$, then $I$ is a prime ideal of $R$.

Proof. If $I$ is a proper strongly divisorial ideal of $R$ and $x \in R \backslash I$, then, by Lemma 1.2 the ideal $\left(I:_{R} x\right)$ is proper and strongly divisorial. We conclude by applying Lemma 1.1 to the set $\mathbf{S}$ of the proper strongly divisorial ideals of $R$.

We observe that the proof of the previous proposition yields the following more general:

Proposition 1.4. For any *-operation, a maximal strong *-ideal is prime.

We now give conditions sufficient for a strongly divisorial ideal to have a strongly divisorial minimal prime. It is known that a prime ideal minimal over a divisorial ideal is a $t$-ideal [10] and that a minimal prime of a strong ideal is strong [11, Proposition 2.1].

Lemma 1.5. If a divisorial ideal I of a domain $R$ contains a power of its radical, then $\sqrt{I}$ is divisorial.

Proof. By assumption, $(\sqrt{I})^{n} \subseteq I$ for some $n \geq 1$. Thus $\left((\sqrt{I})_{v}\right)^{n} \subseteq$ $\left((\sqrt{I})^{n}\right)_{v} \subseteq I$. Hence $(\sqrt{I})_{v} \subseteq \sqrt{I}$ and so $\sqrt{I}$ is divisorial.

Lemma 1.6. If a divisorial ideal $I$ of a domain $R$ is an intersection of primes and the prime $P$ is essential for this representation (that is, it cannot be omitted), then $P$ is divisorial.

Proof. Let $I=\bigcap_{\lambda \in \Lambda} P_{\lambda}$ be a divisorial ideal, where the $P_{\lambda}$ are primes, and let $\mu \in \Lambda$ be such that $J:=\bigcap_{\{\lambda \neq \mu\}} P_{\lambda} \nsubseteq P_{\mu}$. We have $\left(I:_{R} J\right)=$ $\bigcap_{\{\lambda \in \Lambda\}}\left(P_{\lambda}: R J\right)=P_{\mu}$ since, for all $\lambda, J \subseteq P_{\lambda}$ if and only if $\lambda \neq \mu$. It follows that $P_{\mu}$ is divisorial.

Lemma 1.7. Let $\left\{P_{\lambda}\right\}_{\lambda \in \Lambda}$ be a family of prime ideals and let $I=$ $\bigcap_{\lambda \in \Lambda} P_{\lambda}$ be an irredundant intersection. Then the following conditions are equivalent:

(1) I is strong (resp. divisorial);

(2) $P_{\lambda}$ is strong (resp. divisorial) for each $\lambda$.

Proof. By [11, Proposition 3.13], $(R: I)$ is a ring if and only if $\left(R: P_{\lambda}\right)$ is a ring for each $\lambda \in \Lambda$. But, if $J$ is a radical ideal, then $(R: J)$ is a ring if and only if $(R: J)=(J: J)$ [2, Proposition 3.3]. Hence $I$ is strong if and only if $P_{\lambda}$ is strong for each $\lambda$. 
If $P_{\lambda}$ is divisorial for each $\lambda$, then clearly $I$ is divisorial. The converse follows from Lemma 1.6.

Since the radical of a strong ideal is strong [11, Proposition 2.1], an immediate consequence of Lemmas 1.5 and 1.7 is:

Proposition 1.8. If a domain $R$ has a proper strongly divisorial ideal $I$ containing a power of its radical and this radical is an irredundant intersection of primes, then all the minimal primes over I are strongly divisorial.

We now prove that any seminormal domain satisfies the hypothesis that each strongly divisorial ideal is radical. Hence a seminormal domain which has a strongly divisorial ideal that is an irredundant intersection of primes has a strongly divisorial prime.

Recall that a domain $R$ is seminormal if it contains all the elements $x \in K$ such that $x^{2}, x^{3} \in R$.

Lemma 1.9. Let $R$ be a seminormal domain, $T$ an overring of $R$ and $I=(R: T)$. Then $I$ is radical in both $T$ and $R$.

Proof. It is enough to show that $I$ is radical in $T$. Let $t$ be an element of $T$ such that $t^{2} \in I$, thus $t^{2} T \subseteq R$, and $t^{3} T=t^{2}(t T) \subseteq R$. Hence, for any $x \in T$, we have $(t x)^{2}=t^{2} x^{2} \in R$ and $(t x)^{3}=t^{3} x^{3} \in R$. Since $R$ is seminormal, it follows that $t x \in R$ and so $t \in I$.

Lemma 1.9 leads to a characterization of seminormal domains, which is similar to [9, Theorem 1.1].

Proposition 1.10. A domain $R$ is seminormal if and only if each strongly divisorial ideal $I$ of $R$ is radical in the overring $(I: I)$.

Proof. If $I$ is a strongly divisorial ideal of $R$ and $T=(I: I)$, then $I=(R: T)$. Conversely, if $T$ is an overring of $R$ so that $(R: T) \neq 0$, then the ideal $I=(R: T)$ is strongly divisorial and $T \subseteq(I: I)$, Proposition 6].

Then, if $R$ is seminormal and $I$ is strongly divisorial, $I$ is radical in $T=(I: I)$ by Lemma 1.9 ,

Conversely, assume that $R$ is not seminormal and let $x \in K \backslash R$ be such that $x^{2}, x^{3} \in R$. Then the ideal $I=(R: R[x])$ is not radical in $(I: I)$, because $x \in R[x] \subseteq(I: I), x^{2} \in I$ but $x \notin I$.

We will give in the next section examples showing that both the hypotheses of Proposition 1.8 are essential. In fact the examples given in $\S 2.1$ and $\S 2.2$ are non-completely integrally closed, quasilocal onedimensional domains, such that the maximal ideal $M$ is not divisorial. 
In these cases no proper strongly divisorial ideal contains a power of its radical $M$.

On the other hand the example given in $§ 2.3$ is a non-completely integrally closed domain without strongly divisorial prime ideals which can be seminormal (or even integrally closed). In this case, no (radical) strongly divisorial ideal is an irredundant intersection of prime ideals.

We now apply the previous results to obtain other classes of domains that are completely integrally closed if they have no strongly divisorial prime ideals.

Theorem 1.11. Let $R \subsetneq T$ be integral domains with $(R: T) \neq(0)$. If $T$ is Noetherian, then $R$ contains a strongly divisorial prime ideal.

Proof. Let $I=(R: T)$, thus $I$ is a common proper ideal of $R$ and of $T$. Moreover, $I$ is divisorial as an ideal of $R$. Since $T$ is Noetherian, the ideal $I$ of $T$ contains a power of its radical and it has just finitely many minimal primes. Since the radical of $I$ in $R$ is contained in the radical of $I$ in $T$, the ideal $I$ of $R$ contains a power of its radical and it is also an (irredundant) intersection of finitely many primes of $R$. By Proposition 1.8, all minimal primes over $I$ are strongly divisorial.

We note that if the domains $R$ and $T$ satisfy the hypotheses of the previous theorem, then $R$ is a pullback of $T$. Moreover, $T \subseteq R^{*}$.

We now turn to the case of domains with all the maximal $t$-ideals divisorial.

Proposition 1.12. Let $R$ be a domain such that each maximal $t$-ideal is divisorial. If $R$ has no strongly divisorial prime ideals, then $R$ is completely integrally closed.

Proof. Suppose, by contradiction, that $R$ is not completely integrally closed, that is, that in $R$ there is a proper strongly divisorial ideal.

Let $\left\{I_{\lambda}\right\}_{\lambda \in \Lambda}$ be a chain of proper strongly divisorial ideals of $R$. Then the ideal $I=\bigcup_{\lambda \in \Lambda} I_{\lambda}$ is a $t$-ideal and, since each maximal $t$-ideal of $R$ is divisorial, $I_{v}$ is a proper ideal. Moreover,

$$
(R: I)=\bigcap_{\lambda \in \Lambda}\left(R: I_{\lambda}\right)=\bigcap_{\lambda \in \Lambda}\left(I_{\lambda}: I_{\lambda}\right) \subseteq \bigcap_{\lambda \in \Lambda}\left(I: I_{\lambda}\right)=(I: I) .
$$

Hence $I_{v}$ is a strongly divisorial ideal.

It follows that $R$ has a maximal strongly divisorial ideal, which is prime by Proposition 1.3 .

Theorem 1.13. Let $R$ be any domain. The following conditions are equivalent:

(1) $R$ has no proper strong t-ideals; 
(2) $R$ has no strong prime t-ideals;

(3) $R$ has no strongly divisorial prime ideals and each maximal $t$-ideal is t-invertible;

(4) $R$ has no strongly divisorial prime ideals and each maximal $t$-ideal is divisorial;

(5) $R$ is completely integrally closed and each maximal t-ideal is divisorial;

(6) $R$ is a Krull domain;

(7) Each $t$-ideal of $R$ is t-invertible.

Proof. (1) $\Rightarrow(2)$ and (3) $\Rightarrow$ (4) are clear.

$(2) \Rightarrow(3)$ follows from the facts that any $v$-ideal is a $t$-ideal and that a maximal $t$-ideal is either strong or $t$-invertible.

$(4) \Rightarrow(5)$ follows from the Proposition 1.12

$(5) \Rightarrow(6)$ is [7, Corollary 2.8].

$(6) \Rightarrow(7)$ is well known (see for example [7, Corollary 2.7]).

$(7) \Rightarrow(1)$ is clear.

The fact that a domain without strong prime $t$-ideals is completely integrally closed can be also proved recalling that a minimal prime of a proper strongly divisorial ideal is a strong $t$-ideal [11, Proposition 2.1].

A domain in which each $t$-ideal is divisorial is called a $T V$-domain. Examples of $T V$-domains include Mori, in particular Noetherian, domains and pseudovaluation domains 12 .

The following corollary is immediate by Theorem 1.13 and was proved for Mori domains in [3. Corollary 14]; the equivalence $(2) \Leftrightarrow(3)$ in it was also proved in [12, Theorem 2.3].

Corollary 1.14. Let $R$ be any domain. The following conditions are equivalent:

(1) $R$ is a $T V$-domain with no strongly divisorial prime ideals;

(2) $R$ is a completely integrally closed $T V$-domain;

(3) $R$ is a Krull domain.

An invertible maximal ideal is clearly a $t$-invertible maximal $t$-ideal. Hence a domain in which each maximal ideal is invertible satisfies the hypothesis that each maximal $t$-ideal is divisorial. In addition, a completely integrally closed domain in which each maximal ideal is invertible is one-dimensional [7, Theorem 2.1]. Hence we obtain the following results.

Corollary 1.15. Let $R$ be any domain. The following conditions are equivalent: 
(1) $R$ has no strong ideals;

(2) $R$ has no strong prime ideals;

(3) $R$ has no strongly divisorial prime ideals and each maximal ideal is invertible;

(4) $R$ has no strongly divisorial prime ideals and each maximal ideal is divisorial;

(5) $R$ is completely integrally closed and each maximal ideal is divisorial;

(6) $R$ is a Dedekind domain;

(7) Each ideal of $R$ is invertible.

Recall that a $t$-ideal of a domain may be neither strong nor $t$-invertible. However, by Theorem 1.13. if no proper $t$-ideal is strong, then all the $t$-ideals must be $t$-invertible.

Corollary 1.16. Let $R$ be a domain in which each ideal is divisorial. The following conditions are equivalent:

(1) $R$ has no strongly divisorial prime ideals;

(2) $R$ is completely integrally closed;

(3) $R$ is a Dedekind domain.

An integrally closed domain in which each ideal is divisorial is a Prüfer $\sharp \sharp$-domain [6, Theorems 4.3.4 and 4.3.6]. Hence it has the radical trace property [6, Theorem 4.2.28].

Recall that $R$ is a $\sharp \sharp$-domain if each overring $S$ of $R$ has the property that, for each pair of nonempty sets of maximal ideals $\mathcal{M}_{1}$ and $\mathcal{M}_{2}$ of $S, \bigcap_{M \in \mathcal{M}_{1}} S_{M}=\bigcap_{M \in \mathcal{M}_{2}} S_{M}$ only if $\mathcal{M}_{1}=\mathcal{M}_{2}$.

A domain satisfying the radical trace property, or an RTP-domain, is a domain $R$ such that $I(R: I)$ is a radical ideal, for every noninvertible ideal $I$. That is, $R$ is an RTP-domain if and only if each strong ideal of $R$ is radical [6, Section 4.2]. The property that $I(R: I)$ is a prime ideal for every noninvertible ideal $I$, is called the trace property and domains with this property are called TP-domains. Therefore $R$ is a $T P$-domain if and only if each proper strong ideal of $R$ is prime. Examples of $T P$-domains include valuation domains [6, Section 4.2].

It follows directly from the definition that a $T P$-domain without strongly divisorial primes is completely integrally closed.

We do not know whether in general an RTP-domain with no strongly divisorial prime ideals is completely integrally closed. However, the answer to this problem is positive in case $R$ is an $R T P$-domain with the ascending chain condition on radical ideals. In fact, in this case, if the set of maximal strongly divisorial ideals of $R$ is not empty, it has 
maximal elements and these are prime ideals by Proposition 1.3. Recalling that each ideal of a domain with the ascending chain condition on radical ideals has finitely many minimal primes, the same result can also be obtained by applying Lemma 1.6. In addition, it is known that, if $R$ is an $R T P$-domain, then each nonzero nonmaximal prime of $R$ is strongly divisorial [6, Theorem 4.2.16]. Hence an RT P-domain without strongly divisorial primes is always one-dimensional. It follows that a Prüfer $R T P$-domain without strongly divisorial primes is completely integrally closed.

In general a one-dimensional domain without strongly divisorial prime ideals may not be completely integrally closed, as we will show in the next section. The following proposition is a partial positive result:

Proposition 1.17. Let $R$ be a one-dimensional domain that is not completely integrally closed and such that the intersection of the maximal ideals is irredundant (e.g., $R$ has finitely many maximal ideals). If $R$ is either an RTP-domain or seminormal, then it has a strongly divisorial maximal ideal.

Proof. If $R$ is not completely integrally closed, it has a strongly divisorial ideal which is radical (Lemma 1.9). Since this ideal is an irredundant intersection of primes, we conclude by Lemma 1.7.

\section{Counterexamples}

In this Section we give examples of domains which are not completely integrally closed, but do not have strongly divisorial prime ideals.

We denote the integral closure of an integral domain $R$ in its quotient field by $R^{\prime}$.

$\S 2.1$. A one-dimensional non-completely integrally closed quasilocal integral domain $(R, M)$ such that $R^{*}=R^{\prime}$ is also onedimensional quasilocal, $\left(R: R^{*}\right)=(0)$ and such that $M$ is not divisorial.

Let $F$ be a field of finite characteristic $p$ and let $S$ be the additive submonoid of $\mathbb{Q}$ generated by the set of the numbers $\left\{n+1+\frac{1}{2^{n}}\right\}$ for all natural numbers $n \geq 0$. Clearly, $2,5 \in S$. Thus each natural number $n \geq 4$ belongs to $S$.

Consider the monoid domain $F[S]=F\left[X^{S}\right]$, where $X^{S}=\left\{X^{s} \mid s \in\right.$ $S\}$, and set $M=X^{S \backslash\{0\}} F[S]$ and $R=F[S]_{M}$.

For $n \geq 4$, since $n \in S$, then $X^{n} \in R$. In particular this shows directly that $R$ is not seminormal, because $X \notin R$ (cf. Proposition 1.17). 
In order to prove that $R$ has the required properties, we need two Lemmas.

\section{Lemma 2.1.}

(1) For $n \geq 0$, if $m$ is a sufficiently large positive integer, then $\frac{m}{2^{n}} \in S$.

(2) Let $q$ be a rational number. If $q+s \in S$ for each nonzero $s \in S$, then $q \in S$.

(3) If $q$ is a rational number, then $q+\frac{1}{2^{n}} \notin S$ for natural $n \gg 0$.

Proof. (1) For natural $k \gg 0$, the elements $\frac{k 2^{n}}{2^{n}}=k$ and $\frac{k 2^{n}+1}{2^{n}}=$ $(k-n-1)+\left(n+1+\frac{1}{2^{n}}\right)$ belong to $S$ and the numerators of these two fractions are coprime positive integers. Thus, if $m \gg 0$, then $\frac{m}{2^{n}} \in S$.

(2) Let $q=\frac{a}{b}$, where $a$ and $b$ are integers, and $b>0$. Let $k>$ $\max (q, b)$ be an integer. Since $u:=q+k+1+\frac{1}{2^{k}} \in S$, we have $u=\sum_{i=1}^{m}\left(n_{i}+1+\frac{1}{2^{n_{i}}}\right)$ for some natural numbers $n_{1} \leq \cdots \leq n_{m}$, not necessarily distinct. Since $k>q$, we have $n_{i} \geq k$ for at most one index $i$. Since $k>b$, we have $u=\frac{a}{b}+k+1+\frac{1}{2^{k}}=$ $\frac{\alpha}{2^{k} \beta}$, where $\alpha$ and $\beta$ are odd integers. The denominator of $u=\sum_{i=1}^{m}\left(n_{i}+1+\frac{1}{2^{n_{i}}}\right)$ as a reduced fraction is at most $2^{n_{m}}$, thus $n_{m} \geq k$, and $n_{i}<k$ for $i<m$. It follows that $n_{m}=k$ and that $q=\sum_{i=1}^{m-1}\left(n_{i}+1+\frac{1}{2^{n_{i}}}\right) \in S$.

(3) Let $q=\frac{a}{b}$, where $a$ and $b$ are integers, and $b>0$. Let $n>$ $\max (q, b)$ be an integer. Assume that $q+\frac{1}{2^{n}} \in S$, thus $q+\frac{1}{2^{n}}=$ $\sum_{i=1}^{m}\left(n_{i}+1+\frac{1}{2^{n_{i}}}\right)$ for some natural numbers $n_{1} \leq \cdots \leq n_{m}$. Since $2^{n}$ divides the denominator of $q+\frac{1}{2^{n}}$ written as a reduced fraction, we see that $n_{m} \geq n$. Thus $n_{m}+1+\frac{1}{2^{n_{m}}}>n+\frac{1}{2^{n}}>$ $q+\frac{1}{2^{n}}$, a contradiction.

Lemma 2.2. Let $f$ be a nonzero element of $R$. Then, for $n$ sufficiently large, $f^{p^{n}}$ is associated in $R$ with an element of the form $X^{s}$ for some $s \in S$.

Proof. We may assume that $f$ belongs to the ideal $M$ of $F[S]$, so that

$$
f=a_{1} X^{s_{1}}+a_{2} X^{s_{2}}+\cdots+a_{m} X^{s_{m}},
$$

where $s_{1}<\cdots<s_{m}$ are elements of $S$, and the coefficients $a_{1}, \ldots, a_{m}$ are in $F$. We may further assume that $a_{1}=1$. We have

$$
\begin{gathered}
f^{p^{n}}=X^{p^{n} s_{1}}+a_{2}^{p^{n}} X^{p^{n} s_{2}}+\cdots+a_{m}^{p^{n}} X^{p^{n} s_{m}} \\
=X^{p^{n} s_{1}}\left(1+a_{2}^{p^{n}} X^{p^{n}\left(s_{2}-s_{1}\right)}+\cdots+a_{m}^{p^{n}} X^{p^{n}\left(s_{m}-s_{1}\right)}\right) .
\end{gathered}
$$


If $n$ is sufficiently large, by Lemma 2.1 (1), we see that $p^{n}\left(s_{i}-s_{1}\right) \in S$, for $i=2, \cdots, m$ and so

$$
1+a_{2}^{p^{n}} X^{p^{n}\left(s_{2}-s_{1}\right)}+\cdots+a_{m}^{p^{n}} X^{p^{n}\left(s_{m}-s_{1}\right)} \in F[S] .
$$

Hence $f^{p^{n}}$ is associated in $R$ with $X^{p^{n} s_{1}}$.

\section{Proposition 2.3.}

(1) $R$ is one-dimensional.

(2) $R^{\prime}=R^{*}$ is one-dimensional quasilocal.

(3) $\left(R: R^{*}\right)=(0)$.

(4) The ideal $M$ is not divisorial.

(5) $R$ is not integrally closed and has no strongly divisorial prime ideals.

Proof. (1) If $f, g$ are nonzero elements of $M$, then by Lemma 2.2 there exist $m, n \in \mathbb{N}$ such that $f^{m}$ and $g^{n}$ are associated. So $\sqrt{(f)}=\sqrt{(g)}$ and $R$ is one-dimensional (cf. [8, Theorems 17.1 and 21.4]).

(2) By Lemma 2.1 (1) and by [8, Corollary 12.7], we easily obtain that $R^{\prime}=R^{*}=F\left[X^{\frac{m}{n}} \mid m \text { and } n \text { are positive integers }\right]_{N}$, where $N$ is the maximal ideal generated by the elements $X^{\frac{m}{n}}$ for positive integers $m$ and $n$. Hence $R^{*}=R^{\prime}$ is one-dimensional quasilocal.

(3) Suppose that $0 \neq f=a_{1} X^{s_{1}}+a_{2} X^{s_{2}}+\cdots+a_{m} X^{s_{m}} \in\left(R: R^{*}\right)$, where $a_{1} \neq 0$. Then, for $n \gg 0, X^{\frac{1}{2^{n}}} \in R^{*}$, but $f X^{\frac{1}{2^{n}}}=$ $a_{1} X^{s_{1}+\frac{1}{2^{n}}}+\cdots \notin R$, since $s_{1}+\frac{1}{2^{n}} \notin S$ by Lemma [2.1] (3); a contradiction.

(4) To show that $M$ is not divisorial, it is enough to prove that $(R: M) \subseteq R$.

Let $t=\frac{f}{g}$, where $f, g \in F[S]$, such that $t M \subseteq R$. By Lemma 2.2, for $n \gg 0$, the element $g^{p^{n}}$ is associated in $R$ with an element of $X^{S}$. Since $t=\frac{f g^{p^{n}-1}}{g^{p^{n}}}$, we may assume that $t \in F[G]$, where $G$ is the group of quotients of $S$. Since $M=X^{S \backslash\{0\}} R$, it follows that $t^{\prime} M \subseteq M$ for all components $t^{\prime} \in X^{G}$ of $t$. Thus we may assume that $t=X^{q}$, where $q \in G$, thus $q$ is a rational number. Since $t X^{s} \in R$, for each nonzero $s \in S$, by Lemma 2.1 (2) we obtain $t \in R$.

(5) Since, as we have already observed, $R$ is not seminormal, it is certainly not integrally closed. Since $R$ is one dimensional and quasilocal and the unique maximal ideal $M$ is not divisorial, there are no strongly divisorial prime ideals. 


\section{$\S 2.2$. A one-dimensional non-completely integrally closed quasi-} local domain $(R, M)$ such that $R^{*}$ is also one-dimensional quasilocal, $\left(R: R^{*}\right) \neq(0)$, and such that $M$ is not divisorial.

Let $\mathbf{X}=\left\{X_{n}, \mid n \geq 1\right\}$ be a set of indeterminates over a field $F$. We define $w\left(X_{n}\right)=\frac{1}{n}$ (the weight of $X_{n}$ ) for all $n \geq 1$. We extend the function $w$ to the set of monomials in $F[\mathbf{X}]$ setting $w\left(c X_{i_{1}} \ldots X_{i_{m}}\right)=$ $\sum_{j=1}^{m} w\left(X_{i_{j}}\right)$, where $c$ is a nonzero element of $F$ and $X_{i_{1}} \ldots X_{i_{m}}$ are indeterminates in the set $\mathbf{X}\left(i_{1}, \ldots, i_{m}\right.$ not necessarily distinct). For a nonzero polynomial $f \in F[\mathbf{X}]$, we define $w(f)$ to be the least weight of a monomial occurring in $f$. We let $w(0)=\infty$. Clearly, $w(f g)=$ $w(f)+w(g)$ for any polynomials $f$ and $g$. We uniquely extend the function $w$ from $F[\mathbf{X}] \backslash\{0\}$ to a function defined on $F(\mathbf{X})$ and satisfying the above property for rational functions $f$ and $g$. Using the weight $w$ we naturally define a $\mathbb{Q}^{+}$-grading on $F[\mathbf{X}]$ :

$$
F[\mathbf{X}]=\bigoplus_{q \in \mathbb{Q}^{+}} F[\mathbf{X}]_{q},
$$

where $F[\mathbf{X}]_{q}$ is the set of $w$-homogeneous forms of weight $q$, together with 0 , that is, the $F$-linear combinations of monomials of weight $q$ for $q \in \mathbb{Q}^{+}$. If $f=\sum_{i=0}^{r} T_{q_{i}} \in F[\mathbf{X}]$, where $0 \leq q_{0}<q_{1}<\cdots<q_{r}$ are rational numbers, and $T_{q_{0}}, \ldots, T_{q_{r}}$ are $w$-homogeneous forms of weight $q_{0}, \ldots, q_{r}$ respectively, we set $w_{\text {in }}(f)=T_{q_{0}}$ (the $w$-initial form of $f$ ). We let $w_{\text {in }}(0)=0$. Clearly, $w_{\text {in }}(f g)=w_{\text {in }}(f) w_{\text {in }}(g)$ for any polynomials

$f$ and $g$. Thus we may define $w_{\text {in }}\left(\frac{f}{g}\right)=\frac{w_{\text {in }}(f)}{w_{\text {in }}(g)}$ for any nonzero rational function $\frac{f}{g}$, where $f$ and $g$ are nonzero polynomials.

If $q \in \mathbb{Q}$, we denote by $F(\mathbf{X})_{\geq q}$ the set $\{f \in F(\mathbf{X}) \mid w(f) \geq q\}$.

Let $A=F[\mathbf{X}]+F(\mathbf{X})_{\geq 1}$, and let $P$ be the maximal ideal $(\mathbf{X}) F[\mathbf{X}]+$ $F(\mathbf{X})_{\geq 1}$ of $A$. Set $R=A_{P}$, and $M=P A_{P}$, thus $(R, M)$ is a quasilocal domain with quotient field $F(\mathbf{X})$. Moreover:

\section{Proposition 2.4.}

(1) $R^{*}=F(\mathbf{X})_{\geq 0}$ and $\left(R: R^{*}\right) \neq(0)$.

(2) $R$ and $R^{*}$ are quasilocal and one-dimensional, and $R^{*}$ dominates $R$.

(3) The ideal $M$ is not divisorial.

(4) $R$ is not completely integrally closed and has no strongly divisorial prime ideals.

Proof. (1) Clearly $R \subseteq F(\mathbf{X})_{\geq 0}$. The domain $F(\mathbf{X})_{\geq 0}$ is completely integrally closed. Indeed, let $0 \neq f \in F(\mathbf{X})$. If $g f^{n} \in F(\mathbf{X})_{\geq 0}$ for some nonzero $g \in F(\mathbf{X})_{\geq 0}$ and all $n \geq 1$, 
then $w\left(g f^{n}\right)=w(g)+n w(f) \geq 0$ for all $n \geq 1$, which implies that $w(f) \geq 0$. It follows that $f \in F(\mathbf{X})_{>0}$.

Since $X_{1} F(\mathbf{X})_{\geq 0} \subseteq R$, then $F(\mathbf{X})_{\geq 0} \subseteq R^{*}$. Hence $R^{*}=$ $F(\mathbf{X})_{\geq 0}$, and $\left(R: R^{*}\right) \neq 0$.

(2) Clearly, $N:=F(\mathbf{X})_{>0}$ is the unique maximal ideal of $F(\mathbf{X})_{\geq 0}$, and $M:=N \cap R$ is the unique maximal ideal of $R$. If $c_{1}$ and $c_{2}$ are nonzero elements in $N=F(\mathbf{X})_{>0}$, then, for sufficiently large $n$, we have $w\left(\frac{c_{1}^{n}}{c_{2}}\right) \geq 1$. Hence $\frac{c_{1}^{n}}{c_{2}} \in R$. This implies that both $R$ and $F(\mathbf{X})_{\geq 0}$ are one-dimensional since any element in $R \backslash F(\mathbf{X})_{>0}$ is a unit in $R$.

(3) Assume that the maximal ideal $M$ of $R$ is divisorial, i.e. ( $R$ : $M) \neq R$.

Let $h$ be an element in $F(\mathbf{X}) \backslash R$ satisfying $h M \subseteq R$. First we show that $w_{\text {in }}(h) \in F[\mathbf{X}]$. Otherwise we have $w(h)<1$. Let $m$ be a positive integer such that $w(h)+\frac{1}{m}<1$. Choose $n \geq m$ so that $h \in F\left(X_{1}, \ldots, X_{n-1}\right)$. Since $X_{n} \in M$, we have $h X_{n} \in M$, and so there are an element $u \in 1+(\mathbf{X}) F[\mathbf{X}]$ and a polynomial $p \in F[\mathbf{X}]$ such that $u h X_{n}-p \in F(\mathbf{X})_{\geq 1}$. Since $w_{\text {in }}\left(h X_{n}\right)<1$, we obtain that $w_{\text {in }}(p)=w_{\text {in }}\left(u h X_{n}\right)=X_{n} w_{\text {in }}(h)$. Since $h \in F\left(X_{1}, \ldots, X_{n-1}\right)$, we infer that $w_{\text {in }}(h) \in F[\mathbf{X}]$, a contradiction. Hence $w_{\text {in }}(h) \in F[\mathbf{X}]$ as claimed. For some $m$ we have $h \in F\left(X_{1}, \ldots, X_{m}\right)$. Define inductively $h_{0}=h$ and $h_{n+1}=h_{n}-w_{\text {in }}\left(h_{n}\right)$. Clearly, $h_{n} \in F\left(X_{1}, \ldots, X_{m}\right) \backslash R$, $h_{n} M \subseteq R$, and $w\left(h_{n+1}\right)>w\left(h_{n}\right)$ for all $n \geq 0$. It follows that $w_{\text {in }}\left(h_{n}\right) \geq 1$, so $h_{n} \in R$ for $n \gg 0$, a contradiction.

(4) By part (1), $R$ is not completely integrally closed; for example, $\frac{X_{1}}{X_{2}^{2}} \in R^{*} \backslash R$. Further, as in the previous example, since $R$ is one dimensional quasilocal and the unique maximal ideal $M$ is not divisorial, there are no strongly divisorial prime ideals.

\section{§2.3. An infinite dimensional integrally closed non-completely integrally closed domain without strongly divisorial prime ideals such that $\left(R: R^{*}\right) \neq(0)$.}

To construct this example, we use some properties of the domain $E=D\left[Z, \frac{d}{Z}\right]$, where $Z$ is an indeterminate over $D$ and $d \in D$ is a nonzero element. This is isomorphic to the extended Rees Algebra $D\left[d X, \frac{1}{X}\right]$ of the nonzero principal ideal $d D$, via the correspondence $Z \rightarrow \frac{1}{X}$.

We have $\mathrm{Qf}(E)=(\mathrm{Qf}(D))(Z)$ and $D=E \cap \mathrm{Qf}(D)$. 
If $\left(D: D^{*}\right) \neq(0)$, then $E^{*}=D^{*}\left[Z, \frac{d}{Z}\right]$ and $\left(D: D^{*}\right) \subseteq(E:$ $\left.E^{*}\right)$. In fact, since $D^{*}$ is completely integrally closed, then $D^{*}\left[Z, \frac{d}{Z}\right]$ is completely integrally closed [1, Theorem 8] and $D^{*}\left[Z, \frac{d}{Z}\right] \subseteq E^{*}$.

The domain $R$ is constructed in the following way.

Let $A$ be an integral domain such that $A \neq A^{*}$ and $\left(A: A^{*}\right) \neq(0)$, and let $a$ be a nonzero element of $\left(A: A^{*}\right)$.

Let $T_{0}$ and $T_{\varepsilon_{1} \ldots \varepsilon_{n} 0}$, for $n \geq 1$ and $\varepsilon_{1}, \ldots, \varepsilon_{n} \in\{0,1\}$, be independent indeterminates over $A$.

Define inductively $T_{1}=\frac{a}{T_{0}}$, and $T_{\varepsilon_{1} \ldots \varepsilon_{n} 1}=\frac{T_{\varepsilon_{1} \ldots \varepsilon_{n}}}{T_{\varepsilon_{1} \ldots \varepsilon_{n} 0}}$ for $n \geq 1$ and set

$$
R=A\left[T_{\varepsilon_{1} \ldots \varepsilon_{n}} \mid n \geq 1 \text { and } \varepsilon_{1}, \ldots, \varepsilon_{n} \in\{0,1\}\right] .
$$

We have $R=\bigcup_{n=0}^{\infty} R_{n}$, where $R_{0}=A$ and

$$
R_{n}=A\left[T_{\varepsilon_{1} \ldots \varepsilon_{k}} \mid 1 \leq k \leq n \text { and } \varepsilon_{1}, \ldots, \varepsilon_{k} \in\{0,1\}\right]
$$

for each $n \geq 1$.

Moreover the domain $R_{n}$ is obtained from $A$ by constructing iteratively extended Rees algebras of the type $D\left[Z, \frac{d}{Z}\right]$; in fact,

$$
R_{1}=A\left[T_{0}, T_{1}\right]=A\left[T_{0}, \frac{a}{T_{0}}\right]
$$

and, for $n \geq 1$,

$$
R_{n+1}=R_{n}\left[Z_{1}, \ldots, Z_{2^{n}}, \frac{c_{1}}{Z_{1}}, \ldots, \frac{c_{2^{n}}}{Z_{2^{n}}}\right]
$$

where

$$
\left\{Z_{1}, \ldots, Z_{2^{n}}\right\}=\left\{T_{\varepsilon_{1} \ldots \varepsilon_{n} 0} \mid \varepsilon_{1}, \ldots, \varepsilon_{n} \in\{0,1\}\right\}
$$

is a set of algebraically independent elements over $R_{n}$, and

$$
\left\{c_{1}, \ldots, c_{2^{n}}\right\}=\left\{T_{\varepsilon_{1} \ldots \varepsilon_{n}} \mid \varepsilon_{1}, \ldots, \varepsilon_{n} \in\{0,1\}\right\} \subseteq R_{n},
$$

because by definition $T_{\varepsilon_{1} \ldots \varepsilon_{n} 1}=\frac{T_{\varepsilon_{1} \ldots \varepsilon_{n}}}{T_{\varepsilon_{1} \ldots \varepsilon_{n} 0}}$.

Setting $K_{0}=\mathrm{Qf}(A)$ and $K_{n}=\mathrm{Qf}\left(R_{n}\right)$ for $n \geq 1$, we have $K_{n+1}=$ $K_{n}\left(Z_{1}, \ldots, Z_{2^{n}}\right)$ and $\mathrm{Qf}(R)=\bigcup_{n=0}^{\infty} K_{n}$. Since $K_{n} \cap R_{n+1}=R_{n}$, it follows that $K_{n} \cap R=R_{n}$, for all $n \geq 0$.

With this notation, we have:

\section{Proposition 2.5.}

(1) $R^{*}=\bigcup_{n=0}^{\infty} R_{n}^{*}$.

(2) $R$ is not completely integrally closed and $a \in\left(R: R^{*}\right)$. In $\operatorname{particular}\left(R: R^{*}\right) \neq(0)$.

(3) $R$ has no strongly divisorial prime ideals. 
Proof. (1) Let $x \in \mathrm{Qf}(R)$ be almost integral over $R$ and let $d \in R$ such that $d x^{k} \in R$, for all $k \geq 0$. Since $x \in \operatorname{Qf}\left(R_{s}\right)$ and $d \in R_{t}$ for some $s, t \geq 0$, then, for $m$ sufficiently large, $x \in \operatorname{Qf}\left(R_{m}\right)$, $d \in R_{m}$ and $d x^{k} \in R \cap \mathrm{Qf}\left(R_{m}\right)=R_{m}$, for all $k \geq 0$. Hence $x \in R_{m}^{*}$ and $R^{*} \subseteq \bigcup_{n=0}^{\infty} R_{n}^{*}$.

The opposite inclusion is clear.

(2) If $R=R^{*}$, then $A^{*} \subseteq R^{*} \cap \operatorname{Qf}(A)=R \cap \operatorname{Qf}(A)=A$. Since $A$ is not completely integrally closed, neither is $R$.

By hypothesis, $a \in\left(A: A^{*}\right)$ and we have

$$
\left(A: A^{*}\right) \subseteq\left(R_{1}: R_{1}^{*}\right) \subseteq\left(R_{2}: R_{2}^{*}\right) \subseteq \ldots
$$

since $R_{n}$ is obtained from $A$ by constructing iteratively extended Rees algebras of the type $D\left[Z, \frac{d}{Z}\right]$.

To conclude it is enough to observe that

$$
\bigcup_{n=0}^{\infty}\left(R_{n}: R_{n}^{*}\right) \subseteq \bigcup_{n=0}^{\infty}\left(R: R_{n}^{*}\right) \subseteq\left(R: R^{*}\right) .
$$

This follows from (a): $R^{*}=\bigcup_{n=0}^{\infty} R_{n}^{*}$.

Actually, $\left(R: R^{*}\right)=\bigcup_{n=0}^{\infty}\left(R_{n}: R_{n}^{*}\right)$. Indeed, let $y \in(R$ : $\left.R^{*}\right)$. If $y \in \operatorname{Qf}\left(R_{m}\right)$, then $y R_{m}^{*} \subseteq R \cap \operatorname{Qf}\left(R_{m}\right)=R_{m}$. Hence $y \in\left(R_{m}: R_{m}^{*}\right)$ and it follows that $\left(R: R^{*}\right) \subseteq \bigcup_{n=0}^{\infty}\left(R_{n}: R_{n}^{*}\right)$.

(3) Let $P$ be a strongly divisorial prime ideal of $R$. Since $P$ contains $\left(R: R^{*}\right)$, by part $(2), a \in P$.

Since $a=T_{0} T_{1}$, either $T_{0}$ or $T_{1}$ belongs to $P$. We may assume that $T_{0} \in P$ since there is an automorphism of $R$ over $A$ interchanging $T_{0}$ and $T_{1}$. Then, since $T_{0}=T_{00} T_{01} \in P$, either $T_{00}$ or $T_{01}$ belongs to $P$. Since there is an automorphism of $R$ over $A\left[T_{0}\right]$ interchanging $T_{00}$ and $T_{01}$, we may assume that $T_{00} \in P$. Iterating this process, we may assume that all the indeterminates $T_{0}, T_{00}, T_{000} \ldots$ are in $P$. Thus $(R: P) \subseteq(R$ : $\left.\left\{T_{0}, T_{00}, \ldots\right\}\right)$.

We now prove that $\left(R:\left\{T_{0}, T_{00}, \ldots\right\}\right)=R$, thus obtaining a contradiction.

Let $f \in\left(R:\left\{T_{0}, T_{00}, \ldots\right\}\right)$ and let $n \geq 0$ such that $f \in$ $\mathrm{Qf}\left(R_{n}\right)$. As above, we can write

$$
R_{n+1}=R_{n}\left[Z_{1}, \ldots, Z_{2^{n}}, \frac{c_{1}}{Z_{1}}, \ldots, \frac{c_{2^{n}}}{Z_{2^{n}}}\right]
$$

where we can assume that $Z_{1}=T_{0 \ldots 0}$.

Hence $f Z_{1} \in R \cap \operatorname{Qf}\left(R_{n+1}\right)=R_{n+1}$. Since the indeterminates $Z_{1}, \ldots, Z_{2^{n}}$ do not occur in $f$, setting all the $Z_{i}$ 's equal to 1 , we obtain that $f \in R_{n} \subseteq R$. 
To finish, we show in Proposition 2.7 below that this domain can be chosen to satisfy or not either one of the conditions of being seminormal, integrally closed or Archimedean. In the seminormal case the conductor $\left(R: R^{*}\right)$ is a radical strongly divisorial ideal of $R$ (Lemma [1.9), that is not an irredundant intersection of primes (Lemma 1.7).

Lemma 2.6. Let $D$ be an integral domain, $d \in D \backslash\{0\}$ and $E=$ $D\left[Z, \frac{d}{Z}\right]$. Then

(1) $E$ is seminormal if and only if $D$ is seminormal.

(2) $E$ is Archimedean if and only if $D$ is Archimedean.

Proof. (1) Since $D=E \cap Q f(D)$, if $E$ is seminormal, then $D$ is seminormal.

Conversely, assume that $D$ is seminormal and let $f \in Q f(E)$ be such that $f^{2}, f^{3} \in E$. Since $E \subseteq D\left[Z, \frac{1}{Z}\right]$ and $D\left[Z, \frac{1}{Z}\right]$ is seminormal, then $f \in D\left[Z, \frac{1}{Z}\right]$. Write $f=\sum_{i=m}^{n} c_{i} Z^{i}$. Since $D[Z] \subseteq E$, we can assume that $m<0$. We have that $f^{2}=$ $c_{m}^{2} Z^{2 m}+g \in E$. Hence $c_{m}^{2} \in D d^{|2 m|}$ and similarly $c_{m}^{3} \in D d^{|3 m|}$. Since $D$ is seminormal, we obtain that $\frac{c_{m}}{d^{|m|}} \in D$ and so $c_{m} Z^{m} \in$ E.

Repeating this process, we get that all the terms of $f$ are in $E$ and so $f \in E$.

(2) Since $D=E \cap Q f(D)$, if $E$ is Archimedean, then $D$ is Archimedean.

Conversely, assume that $D$ is Archimedean. Let $f(Z), g(Z)$ be two nonzero Laurent polynomials in $E$ such that $g \in \bigcap_{n=1}^{\infty} E f^{n}$. Since the domain $\mathrm{Qf}(D)\left[Z, \frac{1}{Z}\right]$ is Notherian, so Archimedean, we see that $f=c Z^{m}$ for some element $c \in D$ and some integer $m$. Since the coefficients of $g$ belong to $\bigcap_{n=1}^{\infty} D c^{n}$, we see that $c$ is invertible in $D$. If $m \leq 0$, then clearly $f$ is a unit in $E$. If $m>0$, then the coefficients of $g$ belong to $\bigcap_{n=1}^{\infty} D d^{n}$. Hence $d$ is a unit in $D, Z$ is a unit in $E$, and $f=c Z^{m}$ is a unit in $E$. We conclude that $E$ is Archimedean.

\section{Proposition 2.7.}

(1) $R$ is seminormal (resp. integrally closed) if and only if $A$ is seminormal (resp. integrally closed).

(2) $R$ is Archimedean if and only if $A$ is Archimedean. 
Proof. (1) Since $R=\bigcup_{n=0}^{\infty} R_{n}$, it is enough to show that $R_{n}$ is seminormal (resp. integrally closed) if and only if $R_{n+1}$ is seminormal (resp. integrally closed), for each $n \geq 0$.

This follows from the fact that the domain $R_{n+1}$ is obtained from $R_{n}$ by constructing iteratively extended Rees algebras of principal ideals and from the fact that $D\left[Z, \frac{d}{Z}\right]$ is seminormal (resp. integrally closed) if and only if $D$ is seminormal (resp. integrally closed) by Lemma 2.6 (resp. [1, Theorem 8]).

(2) Since $A=R \cap Q f(A)$, if $R$ is Archimedean, then $A$ is Archimedean.

Conversely, assume that $A$ is Archimedean. By Lemma 2.6, all the domains $R_{m}$ are Archimedean.

Now let $r$ be a nonzero element of $R$, and let $c \in \bigcap_{n=1}^{\infty} R r^{n}$. For some $m \geq 0$ we have $r, c \in R_{m}$. Since $\operatorname{Qf}\left(R_{m}\right) \cap R=R_{m}$, we see that $c \in \bigcap_{n=1}^{\infty} R_{m} r^{n}$. Since $R_{m}$ is Archimedean, we conclude that $c=0$ and that $R$ is Archimedean.

\section{REFERENCES}

[1] D. D. Anderson and D. F. Anderson, The ring R $[\mathrm{X}, \mathrm{r} / \mathrm{X}]$, Zero-dimensional commutative rings (Knoxville, TN,1994), 95-113, Lecture Notes in Pure and Appl. Math., 171, Dekker, New York, 1995.

[2] D. F. Anderson, When the dual of an ideal is a ring, Houston J. Math., 9 (1983), 325-332.

[3] V. Barucci, Strongly divisorial ideals and complete integral closure of an integral domain, J.Algebra 99 (1986), 132-142.

[4] V. Barucci and E. Houston, On the prime spectrum of a Mori domain. Comm. Algebra 24 (1996), 3599-3622.

[5] S. Chapman and S. Glaz, Non-Noetherian Commutative Ring Theory, Kluwer Academic Publishers, 2000.

[6] M. Fontana, J. A. Huckaba and I. J. Papick. Prüfer domains, Dekker, 1997.

[7] S. Gabelli, Completely integrally closed domains and t-ideals, Bollettino U.M.I., 3-B (1989), 327-342.

[8] R. Gilmer, Commutative semigroup rings, The University of Chicago Press, 1984.

[9] R. Gilmer and R. Heitmann, On Pic $(R[X])$ for $R$ seminormal, J. Pure Appl. Algebra 16 (1980), 251-257.

[10] F. Halter-Kock, Ideal systems. An introduction to multiplicative ideal theory. Monographs and Textbooks in Pure and Applied Mathematics, 211. Marcel Dekker, 1998.

[11] E. G. Houston, S.-E. Kabbaj, T. G. Lucas and A. Mimouni, When is the dual of an ideal a ring?, J. Algebra 225 (2000), 429-450.

[12] E. G. Houston and M. Zafrullah, Integral domains in which each $t$-ideal is divisorial, Michigan Math. J., 35 (1988), 291-300. 
18 VALENTINA BARUCCI, STEFANIA GABELLI, AND MOSHE ROITMAN

Dipartimento di Matematica, Università di Roma "La Sapienza", PiAZZAle A. Moro, 5, 00185 Roma, ItAly

E-mail address: barucci@mat.uniroma1.it

Dipartimento di Matematica, Università degli Studi Roma Tre, Largo S. L. Murialdo, 1, 00146 Roma, Italy

E-mail address: gabelli@mat.uniroma3.it

Department of Mathematics, University of Haifa, Mount Carmel, HAIFA 31999, ISRAEL

E-mail address: mroitman@math.haifa.ac.il 